\title{
MODELO DE ENTRENAMIENTO PARA LA DETECCION DE TALENTOS DEPORTIVOS EN EL SOFTBOL.
}

\author{
Vera, J. ${ }^{1}$ Flórez. P. ${ }^{2}$ Jaimes, M. ${ }^{3}$
}

1. Vera Jose (2015). Doctor en Ciencias de la Cultura Física, Profesor Universidad del Valle, Grupo de Investigación Grinder, vera.jose@correounivalle.edu.com.

2. Flórez Pedro (2015). Magister en ciencias de la Actividad física y del deporte Profesor Universidad de Antioquia y Universidad San Buenaventura cede Medellín, entrenador INDER Antioquia. pedro.florez @usbmed.edu.co.

3. Jaimes Marco (2015). Doctor en Nuevas Perspectivas de Investigación en la Actividad Física, Profesor Universidad de Pamplona, mafrejala@ugr.es, Grupo de Investigación CTS-642 Universidad de Granada España.

\section{RESUMEN}

La selección de talentos en el softbol ha sido utilizada como estrategia para buscar las élites deportivas, con las cuales se nutren las selecciones de Alto Rendimiento Deportivo de cada país, en ellas se incorporan aquellas personas, normalmente jóvenes en formación, que presentan los rasgos necesarios para rendir de forma sumamente eficiente en la práctica de un deporte. El presente trabajo investigativo se describe en su marco teórico una amplia referencia de conceptos y definiciones de diferentes autores relacionados con la detección de talentos en el softbol. El modelo propuesto se abordó desde la perspectiva de la investigación cuasi experimental, con un grupo experimental, al cual se le aplicaron las pruebas y se sometió a un modelo de entrenamiento, y un grupo control, a cual solo, se le realizaron las pruebas técnicas, la importancia de este trabajo de investigación radica en el modelo novedoso de entrenamiento el cual se fundamenta en tres partes importantes que son; la aplicación de la batería de test, las mediciones antropométricas y la aplicación de la estructura de planificación contemporánea propuesta para tal fin.

Palabras clave: Selección de talentos, softbol, planificación, entrenamiento deportivo.

\begin{abstract}
The talent selection in softball has been used as a strategy to find the sporting elite, with which the High Performance Sports selections each country, including those individuals, usually young are incorporated into training, having the necessary traits are nurtured to pay so highly efficient in practice a sport. This research work is described in its extensive reference framework of concepts and definitions of different authors related to talent identification in softball. The proposed model is discussed from the perspective of the quasi- experimental research with an experimental group, which were applied tests and underwent a training model, and a control
\end{abstract}


group, which only, he performed technical tests the importance of this research lies in the innovative training model which is based on three important parts which are ; applying test battery, anthropometric measurements and the application of the structure of contemporary planning proposal for such purpose.

Keyword: talent selection, softball, planning, sports training.

\section{INTRODUCCIÓN}

La captación de un talento deportivo es una de las tareas más difíciles, polémica y comprometida del entrenamiento con niñas que podamos encontrar.

Es difícil no porque lo sea el hecho en sí mismo de captar un talento, quizás lo más sencillo de todo, sino porque en la actualidad no se considera como un acto único el descubrimiento sino como un proceso continuo y temporal a través del cual surgirá un talento.

Descubrir deportistas con mayor proyección de futuro en el campo del rendimiento, es quizás el problema más importante de los entrenadores actuales. En la promoción y perfeccionamiento de este proceso están |interesados, además de los técnicos y profesores, varias partes. En primer lugar los niños y adolescentes y familias, y en segundo, los clubes, disciplinas y deporte en general (Frenkl, Szabo y Meszaros, 1990).

La escuela Alemana, la soviética, la Checoslovaquia y otros países del este de Europa, se inclinan a la detección precoz de talentos deportivos en el softbol, basados en un modelo global, aunque básicamente establecen las mismas líneas básicas, se suelen diferenciar por poner énfasis en algunos grupos de factores. Los hay los que se apoyan en el desarrollo somático de los jóvenes, poniendo máximo interés en el estudio de estos factores (Ejem, M. (1981), Todorow y Lazarov (182), o autores de países que solo analizan aspectos relacionados con la medicina del deporte, 0 toman las características psicológicas como base de la selección de talentos. Como podemos inferir del modelo europeo, todavía no están definidos los perfiles de exigencias para detección de talentos deportivos en el softbol y ha sido en los últimos años cuando se ha empezado a realizar estudios minuciosos de exigencias específicas de todos los deportes.

La escuela americana, más exactamente los EE.UU, aplica un modelo basado en la performance, el cual, busca establecer la detección a través de los resultados obtenidos, solo se seleccionan los mejores deportistas que han obtenidos los mejores resultados en los test. Claro está, que apoyan su trabajo en un sistema de identificación de talentos deportivos aplicando sistemáticamente y puntualmente unas pruebas de campo y de laboratorio con apoyo de la tecnología. Es importante, manifestar que EEUU es el país que mejor resultados deportivos ha obtenido a nivel mundial en el softbol. Esto, se debe al modelo científico debidamente estructurado y de una aplicación sistemática, para la detección de talentos deportivos en el softbol, con el fin último, de conseguir los objetivos marcados en un trabajo a medio y largo plazo, y más hoy en que el deportista de alta competición se le exige esfuerzos excepcionales.

La escuela Cubana, fundamenta su modelo en la llamada "Pirámide Deportiva del Alto rendimiento". El éxito de este modelo está en los resultados obtenidos, y que radica básicamente en una perfecta coordinación de todas las entidades y administraciones que intervienen en el ámbito deportivo. De 
ahí que; Cuba es potencia en softbol, que sus deportistas hacen un proceso desde la escuela, la iniciación deportiva, prospecto deportivo, talento deportivo y para llegar a la Excelencia deportiva.

En países sudamericanos (Brasil y Argentina preferentemente) fundamentan su trabajo bajo los parámetros del modelo de "Selección por traslado de talentos y entrenamiento". El cual, consiste en seleccionar a las niñas que han completado su ciclo de crecimiento en su práctica totalidad (17-18 años) y que destaca en sus performances de capacidades más definitorias en el softbol. Una vez detectada y seleccionada, se pasa a un entrenamiento intensivo.

En Colombia, la detección de talentos deportivos en el softbol, se basa en un modelo de competición y selección, el cual se realiza en el transcurso de las competiciones regulares, normalmente a través de un seguimiento por observadores calificados, pasando posteriormente a formar parte de los escalones inferiores de los clubes, controlando la progresión de los deportistas para ir pasando a los equipos de mayor categoría. Este modelo, consiste en designar entrenadores de softbol, que se desplacen a las regiones, para que observen todas las competiciones de cierto nivel, en categorías jóvenes, para detectar talentos deportivos, con el fin de formar parte de las selecciones juveniles, sub 23 y mayores de softbol de Antioquia.

\section{MÉTODO}

El universo de la población en esta investigación fue 1200 niños, niñas y jóvenes, la cual constituyó la matricula inicial de los deportistas con edades comprendidas entre los 6 y 21 años, que hacen parte de las Escuelas Populares del Deporte, de los programas de formación deportiva de las diferentes comunas y veredas del Municipio.
Como un subgrupo de la población. Para lo cual se tomó una muestra aleatoria de 20 niñas de sexo femenino, con edades entre los 14 y 15 años. De las cuales, 10 niñas conforman el grupo experimental y 10 niñas el grupo de control, las cuales realizan entrenamiento durante 3 veces a la semana con una duración de 2 horas en el estadio de Softbol Oswaldo Osorio. Esta muestra se determinó bajo el criterio de inclusión, que estableció la modalidad de formación deportiva que las niñas practican (Softbol), que tuvieran instalaciones deportivas y espacios donde se garantizara la aplicación con calidad de la batería de pruebas a desarrollar.

\section{DESARROLLO Y EXPLICACIÓN DE LAS PRUEBAS}

Test físicos: a continuación se enumeran las diferentes pruebas físicas aplicadas:

Prueba No1: Abdominales en 30 segundos: Objetivo: Medir la fuerza-resistencia de los músculos lumbo-abdominales.

Ejecución: El deportista se acuesta sobre la espalda, con las piernas flexionadas, los pies de planta sobre el suelo y los brazos cruzados sobre el pecho con las manos en los hombros. Un compañero sujeta firmemente los pies contra el suelo, que estarán de 30 a $45 \mathrm{cms}$ de los glúteos, evitando que se despeguen del mismo. A la señal de "listos" - "ya", el deportista se sienta y toca con sus codos los muslos; inmediatamente retorna a la posición inicial y continua repitiendo el ejercicio hasta que el entrenador le diga "alto", justamente un minuto después de haber sido iniciado.

Material: Campo al aire libre (césped) y cronómetro.

Anotación: Se anota el total de repeticiones realizadas correctamente.

Prueba No2: Salto Horizontal sin impulso (Pie juntos). 
Objetivo: Medir la fuerza explosiva.

Ejecución: El deportista se sitúa detrás de la línea de carrera de 1era base, con los pies juntos, balanceándose varias veces a hacia adelante y hacia atrás con ayuda de sus brazos se impulsa y salta hacia adelante cayendo con los pies juntos.

Material: Campo de softbol - Línea de carrera de 1era Base y cinta métrica.

Anotación: Se realizan tres saltos y se toma el mayor.

Prueba No3: Lanzamiento del balón medicinal (2kgs).

Objetivo: Medir la fuerza de los miembros superiores.

Ejecución: El deportista se sitúa detrás de la línea de carrera de 1era base, con el pie derecho ligeramente adelante del pie izquierdo, y con el balón medicinal (2 kgms) sostenido con sus dos manos, para lo cual se le permite balancear varias veces a hacia adelante y hacia atrás sus brazos, realizando un lanzamiento por encima de la cabeza lo más lejos posible.

Material: Campo de softbol - Línea de carrera de 1era Base, balón medicinal de 2 Kgms y cinta métrica.

Anotación: Se realizan tres lanzamientos y se toma el mayor.

Prueba No4: Volante HOME - Primera Base sin impulso.

Objetivo: Medir la velocidad del deportista del home a primera base.

Ejecución: El deportista se sitúa en la caja de bateo y realiza swing simulado y corre por la línea de carrera hacia la 1era base, la cual pisa y sigue hacia el terreno de faul. En la 1era base se sitúa el evaluador y a la señal de listo realiza el swing y cuando pisa la base se registra el tiempo alcanzado.
Material: Campo de softbol - Línea de carrera de 1era Base, cronometro y Bate de sóftbol.

Anotación: se realizan tres intentos y se toma el mejor tiempo

Pruebas antropometricas:

El análisis y la interpretación de las medidas antropométricas permiten conocer el estado de crecimiento y desarrollo, el estado nutricional como también aptitudes $y$ cualidades para el desarrollo de alguna actividad física.

El estudio antropométrico comprende la composición corporal y el somatotipo. La composición corporal analiza la estructura, proporcionalidad y las relaciones existentes entre los diferentes constituyentes del cuerpo humano.

Masa corporal: es el criterio más comúnmente empleado para enjuiciar el grado de sobrepeso u obesidad, no es necesariamente el más exacto. La obesidad debe entenderse como un exceso de grasa y no simplemente como un exceso de peso total. Cabe anotar que existen modalidades deportivas y categorías en las cuales es importante un alto volumen de peso global y graso. Por ejemplo: zumo, judo, halterofilia, entre otros. Para calcular el peso de las niñas evaluadas se utilizó una balanza digital.

Talla: es un indicador que señala el crecimiento longitudinal 0 lineal, fundamentalmente del tejido óseo. Para calcular la estatura se utilizó un tallímetro. Características que debe reunir:

Una superficie vertical fija, rígida, con una escala graduada en centímetros, adherida a la misma. Una superficie horizontal móvil, en ángulo recto con la superficie vertical, que se deslice libremente hacia arriba y hacia abajo. 
Plano inferior constituido por el suelo o cualquier plano de apoyo sobre él, que conocida con el = de escala de medición.

Pliegues de tejido adiposo: esta medición se emplea para determinar el porcentaje y el peso graso del individuo. Es quizás la variable más importante en el estudio de composición corporal ya que permite conocer, con muy buena aproximación, la realidad antropométrica de las personas evaluadas. Existen diferentes métodos, técnicas, puntos anatómicos e instrumentos para determinar el porcentaje de grasa corporal. Se conocen cerca de 20 puntos en los cuales se pueden tomar los pliegues de tejido adiposo. Se recomienda tomar entre 4 y 8 pliegues de tejido adiposo para determinar el porcentaje de grasa corporal.

Entre las técnicas más comúnmente empleadas están la Densitometría, la impedancia eléctrica y la Medición de Pliegues Cutáneos, siendo esta última la más frecuentemente aplicada, ya que es menos costosa y requiere elementos de fácil adquisición y manejo.

El método de Yuhazz, fue el utilizado, para determinar el porcentaje de grasa por medición de 6 pliegues de tejido adiposo (tríceps, subescapular, abdominal, suprailiaco, muslo anterior y pierna media interna). El cual, hace diferenciación para hombres y mujeres, deportistas y no deportistas.

Método Yuhazz para calcular \% graso féminas deportistas. El instrumento empleado para medir el pliegue de grasa fue el Adiptómetro Harpenden (John Bull).

Índice de masa corporal de Quetelet

La fórmula para calcular índice masa corporal es: IMC $\mathrm{Kg} / \mathrm{m}^{2}=$ PESO/TALLA ${ }^{2}$. El IMC corporal deseable oscila entre 20-19 Kilogramos sobre metro.

\begin{tabular}{|l|l|l|l|l|}
\hline Sexo & Bajo & Deseable & $\begin{array}{l}\text { Sobre } \\
\text { peso }\end{array}$ & Obesidad \\
\hline Hombre & 20 & $20-25$ & $25-30$ & 30 \\
\hline Mujer & 19 & $19-24$ & $24-30$ & 30 \\
\hline
\end{tabular}

Test tecnicos pedagogicos: a continuación se esbozan las pruebas específicas para el softbol:

Técnica individual general:

Manera de agarrar la pelota:

Dedos que intervienen, acción de las yemas y defectos más comunes en el agarre de la pelota.

Maneras de lanzar la pelota:

En péndulo, - Sobre el hombro, - De costado, - De costado por debajo de la cintura.

Estudio de los desplazamientos del brazo, el cuerpo y de las piernas, antes, durante y después de jugar la pelota.

Maneras de recibir la pelota: - De un pase 0 lanzada por un compañero, - De aire (flay), puede ser de frente, de lado o de espalda Por el suelo (roletazo).

Forma correcta de batear la pelota: Formas de tomar el bate, importancia de la colocación de las manos, Colocación de los jugadores en la caja, posición de los pies, de las piernas, del tronco, de los brazos y del bate, Como golpear, acción de las piernas, movimiento de la cadera y el

\begin{tabular}{|c|l|c|}
\hline SEXO & DEPORTISTAS & NO DEPORTISTAS \\
\hline FEMINAS & $\begin{array}{l}\sum 6 \mathrm{PL}(0.1548)+ \\
3.5803\end{array}$ & $\sum 6 \mathrm{PL}(0.1429)+4.56$ \\
\hline \multicolumn{2}{|l|}{ tronco, desplazamiento de los brazos }
\end{tabular}
terminación del movimiento e iniciación de la carrera.

El "Swing", su importancia, procedimientos más usuales, momento oportuno para su 
ejecución, dirección que debe imprimirse a la pelota. Dominio del bateo: dirección, control y ubicación.

Maneras de correr las bases: Cómo correr a una base: salida, pique, trayectoria. Cómo correr más de una base, recorrido, posición del cuerpo. Cómo pisar las bases: importancia de no frenarse, lugar en que se debe pisar, posición del pie sobre la base. Cómo arrojarse a las bases: deslizamientos de costado, de espalda y de frente.

Técnica individual especial.

Lanzador: Técnicas de los lanzamientos: cómo tomar la pelota, presentación y posición correcta en la caja. Tipos de lanzamientos, lentos, rápidos, Curvas, Drop y slider: control de las mismas.

Desplazamientos en la placa.

Receptor: Técnica de la recepción Posición de las piernas, colocación de las manos ubicación en la caja. Técnicas de la defensa: dirección del equipo, entendimiento con el lanzador, señas, colaboración con el primera y tercera base, robo de bases.

Primera base: Características individuales: ubicación en el campo, colocación de los pies y el cuerpo, radio de acción. Colaboración con el segunda base. Rapidez física y mental para realizar dobles y triples juegos.

Segunda base: Característica individual: ubicación en el campo, colocación de los pies y el cuerpo, radio de acción. Colaboración con el Short stop y jugar de intención con el receptor.

Tercera base: Característica individual: ubicación en el campo, colocación de los pies y el cuerpo, radio de acción. Apoyo con el Short stop y los out filders.
Short stop: Característica individual: ubicación en el campo, colocación de los pies y el cuerpo, radio de acción. Colaboración con el segundo base $y$ asistencias a los out filders.

Jugadores de campo: Su importancia, velocidad en los desplazamientos, rapidez y potencia en los lanzamientos, ejercitación para la recepción de las pelotas de aire.

Desplazamientos en el campo y cubrimiento de bases.

Técnicas de conjunto.

Técnica ofensiva: turno de bateo, importancia del orden de bateo y la orientación del mismo. Cuando se debe batear y cuando no el primer lanzamiento con/sin corredor en la primera base.

Esperar cuatro malas.

Bateo de sacrificio.

Ejercicios ofensivos: Carrera de doble posible triple con coach, Salir al robo de 3ra base, Salir para 2da en Hit and Run, Salir al zqueese play con lanzador y sin bateador, Reglas, Juego.

Técnica defensiva: distribución de los jugadores en el campo. Desplazamientos, cubrimientos, zonas de acción de cada jugador. Asistencia. Jugadas forzadas. Tomar-pisar. Tomar-tocar. Dobles-triples jugadas.

Juegos de ejercitación y aplicación.

Habilidades defensivas:

Formación contra el sacrificio con corredor en 1ra base y en 2da base, Formación contra el doble robo Además, se realizaran los siguientes test técnicos pedagógicos que nos permitirán evaluaran las habilidades especificas del softbol: 
Mecánica de lanzamiento: se evalúa la técnica del tiro a las bases por encima del brazo, de lado y bombeado, después de recibir por parte del docente evaluador.

Medición: Para cada evaluación se dará un puntaje de 1 a 4 y se suman los totales en cada gesto técnico.

Material: campo de juego e implementos de juego: bolas y manillas.

Fildeo de flay, rolling y tiro a las bases: se evalúa el gesto técnico del fildeo de bolas de rolling y flay, como se ubica el cuerpo, la manilla y el paso al momento de tirar las bolas las bases.

Medición: Para cada evaluación se dará un puntaje de 1 a 4 y se suman los totales en cada gesto técnico.

Material: campo de juego e implementos de juego: bolas, bate de softbol y manillas.

Mecánica del bateo: se evalúa la ejecución correcta del swing al momento de impactar la bola, la correcta ubicación en la caja de bateo, el agarre del bate, el tiempo y distancia con el contacto con el implemento de juego.

Medición: Para cada evaluación se dará un puntaje de 1 a 4 y se suman los totales en cada gesto técnico.

Material: campo de juego e implementos de juego: bolas y bate de softbol.

Asistencias: se evalúa la correcta ubicación del softbolista en el momento de asistir los tiros o lanzamientos de los compañeros a las almohadillas en las situaciones de robo y Corren de bases.

Medición: Para cada evaluación se dará un puntaje de 1 a 4 y se suman los totales en cada gesto técnico.
Material: campo de juego e implementos de juego: bolas, base, bate de softbol y manillas.

Estructura de planificación del modelo

Para el desarrollo del modelo se utilizó la planificación del proceso de preparación deportiva través de los problemas de dirección de la preparación, la metodología y aspectos prácticos de la programación, la periodización del proceso teniendo en cuenta las indicaciones metodológicas establecidas por Zhelyazkov, T. (2006), en el libro "Teoría y metodología del entrenamiento deportivo" en donde realiza un estudio profundizado de las diferentes formas de planificar el proceso de enseñanza y entrenamiento. Teniendo en cuenta las tendencias contemporáneas del entrenamiento deportivo se construyó un plan de enseñanza a través de modelos de macro estructura, Mesociclos, Microciclos y sesiones de entrenamiento.

Las nuevas tendencias de la planificación del entrenamiento deportivo nos orientan a realizar dosificaciones de la carga teniendo presente los ciclos pequeños los cuales se elaboran a través de Mesociclos, Microciclos y unidades de entrenamiento, su elaboración debe ir directamente hacia la clasificación del contenido de la preparación técnico-táctica del softbol.

Esta investigación tuvo una intervención pedagógica de 4 meses en la cual se realizaba constantemente retroalimentación y formación investigativa de más de dos años, tiempo suficiente para aplicar el método científico en el objetivo planteado. Véase en el siguiente cuadro el modelo de intervención. 


\begin{tabular}{|c|c|c|c|c|}
\hline MESES & AGOSTO & SEPTIEMBRE & OCTUBRE & NOVIEMBRE \\
\hline \multicolumn{5}{|l|}{ MACROCICLOS } \\
\hline PERIODOS & 1 & 2 & 2 & 2 \\
\hline ETAPAS & 2 & 4 & 4 & 4 \\
\hline MESOCICLOS & 1 & 2 & 3 & 4 \\
\hline $\mathrm{N}^{\circ}$ DE MESOCICLOS & 1 & 1 & 1 & 1 \\
\hline FECHA INICIO MESOCICLO & 3 agosto & 31 agosto & 28 septiembre & 26 octubre \\
\hline FECHA FINAL MESOCICLO & 28 agosilo & 25 septiembre & 23 octubre & 20 noviembre \\
\hline $\begin{array}{lcl}\text { DIAS } & \text { Y } & \text { SESIONES } \\
\text { MICROCICLOS } & \end{array}$ & 3 & 3 & 3 & 3 \\
\hline HORAS POR SESION & 1.5 & 1.5 & 1.5 & 1.5 \\
\hline HORAS TOTAL POR MICRO & 4.5 & 4.5 & 4.5 & 4.5 \\
\hline TEST PEDAGOGICOS & 1 & - & $\cdot$ & 1 \\
\hline PRUEBAS DE CONTROL & 2 & - & $\cdot$ & - \\
\hline VOLUMEN TOTAL (TIEMPO) & 18 & 18 & 18 & 18 \\
\hline $\begin{array}{l}\text { TECNICA OFENSIVA - BATIN } \\
\text { CORRIDO }\end{array}$ & 16 & 14 & 14 & - \\
\hline $\begin{array}{l}\text { TECNICA OFENSIVA - ORDEN } \\
\text { AL BATE }\end{array}$ & 8 & - & $\cdot$ & $\cdot$ \\
\hline $\begin{array}{l}\text { TECNICA OFENSIVA - ROBO } \\
\text { DE BASES }\end{array}$ & 4 & 14 & 14 & $\cdot$ \\
\hline $\begin{array}{l}\text { TECNICA DEFENSIVA - } \\
\text { CUADRO CERRADO }\end{array}$ & $\cdot$ & $\cdot$ & $\cdot$ & 14 \\
\hline $\begin{array}{l}\text { TECNICA DEFENSIVA- } \\
\text { DOBLE PLAY POR 2da base }\end{array}$ & $\cdot$ & $\cdot$ & $\cdot$ & 7 \\
\hline $\begin{array}{l}\text { TECNICA DEFENSIVA - } \\
\text { DOBLE PLAY por 3era base }\end{array}$ & $\cdot$ & - & $\cdot$ & 7 \\
\hline $\begin{array}{l}\text { PREPARACION FISICAY } \\
\text { MOTRIZ }\end{array}$ & 4 & 4 & 4 & 4 \\
\hline TEST PEDAGOGICOS & 1 & $\cdot$ & $\cdot$ & 1 \\
\hline PRUEBAS DE CONTROL & 1 & - & - & 1 \\
\hline
\end{tabular}

\section{RESULTADOS}

Pruebas fisicas y tecnicas del grupo experimental

\section{Pruebas antropometricas}

\begin{tabular}{|c|c|c|c|c|c|}
\hline INDIVIDUO & EDAD & PESO & TALLA & IMC & SCLY PLLEGUES en mm \\
\hline 1 & 14 & 45,5 & 1,65 & $|6,7|$ & 15,65 \\
\hline 2 & 15 & 40,6 & 1,48 & 18,54 & 18,13 \\
\hline 3 & 15 & 52 & 1,52 & 22,51 & 19,52 \\
\hline 4 & 15 & 37,6 & 1,57 & 23,37 & 22,77 \\
\hline 3 & 13 & 50,3 & 1,6 & 19,65 & 19,83 \\
\hline 6 & 14 & 54,1 & 1,46 & 25,38 & 24,32 \\
\hline 1 & 15 & 51,7 & 1,6 & 20,20 & 15,96 \\
\hline 8 & 15 & 62,4 & 1,55 & 25,97 & 25,87 \\
\hline 9 & 15 & 60,3 & 1,59 & 23,85 & 20,91 \\
\hline 10 & 15 & 49 & 1,68 & 17,36 & 16,42 \\
\hline PROMEDIOS & 14,6 & 52,35 & 1,57 & 21,35 & 19,94 \\
\hline DES MEDDA & & $6(2) 2$ & 1007 & 3060 & \\
\hline VARIANZA & & 43,98 & 0,00 & 1098 & \\
\hline
\end{tabular}

\begin{tabular}{|r|r|r|}
\hline Z PESO & Z TALLA & \multicolumn{1}{|c|}{ Z IMC } \\
\hline$-1,0$ & $-1,5$ & $-1,5$ \\
\hline 2,9 & $-1,3$ & $-0,9$ \\
\hline$-0,1$ & $-0,7$ & 0,4 \\
\hline 0,8 & 0,0 & 0,7 \\
\hline$-0,3$ & 0,4 & $-0,6$ \\
\hline 0,3 & $-1,6$ & 1,3 \\
\hline$-0,1$ & 0,4 & $-0,4$ \\
\hline 1,5 & $-0,3$ & 1,5 \\
\hline 1,2 & 0,3 & 0,8 \\
\hline$-0,5$ & 1,6 & $-1,3$ \\
\hline
\end{tabular}

Se aplica un procedimiento de comparación múltiple para determinar las medias que son significativamente diferentes unas de otras. La mitad inferior de la salida muestra la diferencia estimada entre cada para de medias.

No hay diferencias estadísticamente significativas entre ningún par de medias a un nivel de confianza.95.0\%.

El cuarto estadístico mostrado en esta tabla, comprueba la hipótesis nula de que la desviación típica de SUMA DE PLIEGUES en $\mathrm{mm}$ dentro de cada uno de los 2 niveles de Grupo, es la misma. De particular interés están los tres $p$-valores. Dado que el menor de los $p$-valores es superior o igual a 0.05 , no hay diferencia estadísticamente significativa entre las desviaciones típicas para un nivel de confianza del $95.0 \%$.

Pruebas físicas

\begin{tabular}{|r|r|r|r|}
\hline ABD 30 Sg. & LAN. BALON 3 KGS en m. & VOL HOME 1B en Sg. & SALTO HTAL \\
\hline 24 & 4,10 & 3,35 & 1,69 \\
\hline 23 & 4,46 & 3,45 & 1,7 \\
\hline 28 & 4,94 & 3,75 & 1,78 \\
\hline 23 & 4,36 & 4,1 & 1,6 \\
\hline 24 & 4,30 & 3,48 & 1,56 \\
\hline 28 & 4,35 & 3,72 & 1,39 \\
\hline 27 & 5,19 & 3,3 & 1,93 \\
\hline 21 & 5,35 & 3,66 & 1,67 \\
\hline 27 & 6,00 & 3,57 & 1,52 \\
\hline 23 & 3,98 & 3,53 & 1,65 \\
\hline$X=24,8$ & $X=4,70$ & $X=3,59$ & $X=1,65$ \\
\hline
\end{tabular}

El cuadro estadístico mostrado en esta tabla, comprueba la hipótesis nula de que la desviación típica de ABD 30 SEG dentro de 
cada uno de los 2 niveles de Grupo, es la misma. De particular interés están los tres $p$-valores. Dado que el menor de los pvalores es superior 0 igual a 0.05 , no hay diferencia estadísticamente significativa entre las desviaciones típicas para un nivel de confianza del $95.0 \%$.

El test de Kruskal-Wallis prueba la hipótesis nula de igualdad de las medianas de LAN_ BALON 3 KGS en mts dentro de cada uno de los 2 niveles de Grupo. Los datos de todos los niveles primero se combinan y se ordenan de menor a mayor. Luego se calcula el rango medio para los datos en cada nivel. Puesto que el $p$-valor es inferior a 0.05 , hay diferencia estadísticamente significativa entre las medianas a un nivel de confianza del $95.0 \%$.

Pruebas técnicas: pretest y post

\begin{tabular}{|r|r|}
\hline $\begin{array}{l}\text { PRETEST } \\
\text { TECNICOS }\end{array}$ & $\begin{array}{l}\text { POSTTEST } \\
\text { TECNICOS }\end{array}$ \\
\hline 30 & 32 \\
\hline 23 & 28 \\
\hline 32 & 32 \\
\hline 31 & 31 \\
\hline 30 & 30 \\
\hline 33 & 33 \\
\hline 35 & 33 \\
\hline 33 & 33 \\
\hline 32 & 32 \\
\hline 23 & 32 \\
\hline$X=30,2$ & $X=31,6$ \\
\hline
\end{tabular}

Promedio Pre test $=30,2$

Promedio Pos test $=31,6$

La tabla ANOVA descompone la varianza de VOLANTE HOME 1B en segundos en dos componentes: un componente entre grupos y un componente dentro de los grupos. El F-ratio, que en este caso es igual a 11.3633 , es el cociente de la estimación entre grupos y la estimación dentro de los grupos. Puesto que el $p$-valor del test $F$ es inferior a 0.05 , hay diferencia estadísticamente significativa entre las VOL
HOME $1 \mathrm{~B}$ en seg medias de un nivel de Grupo a otro para un nivel de confianza del $95.0 \%$. Para determinar las medias que son significativamente diferentes unas de otras, seleccione los Tests de Rangos Múltiples.

El cuarto estadístico mostrado en esta tabla, comprueba la hipótesis nula de que la desviación típica de VOL HOME 1B en seg dentro de cada uno de los 2 niveles de Grupo, es la misma. De particular interés están los tres $p$-valores. Dado que el menor de los $p$-valores es superior o igual a 0.05 , no hay diferencia estadísticamente significativa entre las desviaciones típicas para un nivel de confianza del $95.0 \%$.

\section{DISCUSIÓN}

La implementación de la propuesta metodológica en el grupo intervenido, lo que demuestra que utilizando los medios y actividades propias del entrenamiento deportivo, se logran resultados positivos en las capacidades físicas en jugadoras de softbol, convirtiéndose la propuesta en un mecanismo útil para la detección de talentos en este deporte. Este resultado, concuerda con lo encontrado por (Doniesky Ortiz \& Lescano Chavez, Sf), quienes demostraron que un programa de ejercicios físicos, es eficaz a la hora de encontrar y seleccionar talentos deportivos.

También se encontró que en todas las pruebas físicas hay diferencias altamente significativas en el Post test técnico con respecto al Pret test técnico, entre el Grupo Experimental y el Grupo Control $(p<0.05)$, este resultado, también concuerda con lo hecho por (Doniesky Ortiz \& Lescano Chavez, Sf), quienes también encontraron luego de la aplicación de que su programa de ejercicios, que se mejoró el rendimiento físico de las nueve atletas intervenidas.

Por otro lado, desde el punto de vista pedagógico, la intervención fue aportante en el estudio, pues las atletas intervenidas obtuvieron aprendizajes que se vieron 
reflejados en su rendimiento físico, lo que significa que los planes y métodos globales aplicados se ajustaron adecuadamente al grupo experimental durante varias sesiones de entrenamiento; Esto apoya lo expuesto por Harre (1975), quien en su descripción de los principios del entrenamiento, los Pedagógicos, son aquellos que responden a una concepción metodológica y que su aplicación requiere pautas didácticas para alcanzar los objetivos, es decir, se destaca la importancia de la didáctica y la pedagogía como elementos claves a la hora de implementar estrategias de entrenamiento deportivo, en este caso para la selección de talentos.

Finalmente, en el contexto de este estudio se mostró que con la aplicación de test técnicos se puede identificar y detectar talentos de manera científica, lo que contribuye a mejorar el proceso de selección de deportistas con niveles significativos de juego. Se pretende entonces avanzar a procesos más objetivos en la detección del talento y con una aplicabilidad científica, evitando el empirismo sólo a través de la observación. En esta selección es fundamental el papel protagónico de la escuela, la cual como opina Alfaro (Alfaro, 2004) "la sensibilización de la escuela y la formación de los docentes para la identificación y detección temprana de niños y niñas con altas capacidades motrices".

En pocas palabras la identificación y selección de talentos deportivos es una acción colectiva, entre docentes y entrenadores, que cobija a diferentes instituciones sociales $y$ entre ellas principalmente la escuela y los entes deportivos, que son quienes deben tener los recursos y herramientas metodológicas para tal fin, como por ejemplo la que se propone con ese trabajo.

\section{CONCLUSIONES}

El principal resultado de esta investigación, es que la aplicación de la propuesta metodológica es estadísticamente apropiada para la detección de talento en el softbol, por lo tanto los planes y métodos globales aplicados se ajustaron adecuadamente a las atletas que hicieron parte del grupo experimental durante varias sesiones de entrenamiento, hecho que sugiere la promoción y replicación de la misma para grupos similares; desde el punto de vista pedagógico, la intervención muestra como la reflexión del entrenador, manifiesta en la selección de adecuados planes y métodos globales que se ajustan a las características del grupo intervenido genera resultados objetivos y aplicables en el ámbito del entrenamiento deportivo, específicamente en la detección y selección de talentos.

Al final, con este estudio, se muestra como los test técnicos son una herramienta útil en el proceso de identificar y detectar talentos de manera científica, lo que contribuye significativamente a la objetividad y efectividad del proceso.

\section{REFERENCIAS BIBLIOGRÁFICAS}

Alfaro, É. (2004). El talento psicomotor y las mujeres en el deporte de alta competición. Revista de educación (335), 127-151.

Alexander, Pedro. (1995). "Aptitud Física, Características Morfológicas y Composición Corporal. Pruebas Estandarizadas en Venezuela". Impreso por Graficas Reus. Primera edición. Caracas, Venezuela

Bloom, B.S. (1995). "Developing talent in youg people". NEW YORK, Ballantine.

Bompa, T. (1987). La selección de atletas con talento. Entrenamiento Deportivo. Vol. I. $n^{\circ} 2,46-54$.

Dadan Muñoz, (2005) "Silvana. Antropometría. Generalidades y aplicaciones. Módulo de Nutrición y 
Dietética". Pontificia Universidad Javeriana. Bogotá, Colombia.

Doniesky Ortiz, G., \& Lescano Chavez, A. (Sf). Monografías. Recuperado el 20 de Julio de 2011, de http://www.monografias.com/trabajos82/ren dimiento-deteccion-talentos-softballfemenino/rendimiento-deteccion-talentossoftball-femenino.shtml

Federación Internacional de Softbol. (2007) Documento Sóftbol escuelas. Página 3.

Fetz, F (1998) "Test Deportivos Motores". Editorial KAPELUSZ. S. A. Buenos aires, Argentina, Pág. 18 y 19.

Gutiérrez, A. (1991). Bases para una correcta detección del talento deportivo (I). El entrenador español, $n^{\circ}$ 49, 43-47.

Gutiérrez, A. (1991). Bases para una correcta detección del talento deportivo (II). El entrenador español, $n^{\circ} 50,35-39$.

Indeportes Antioquia. (2005). Plan de Desarrollo, "Por la recuperación del liderazgo deportivo".

Leiva Rodriguez, J., \& García, D. (Sf). Recuperado el 10 de Julio de 2011, de http://50.97.140.160/PubliCE/Articulo.asp?id $a=331$

Lopez B, Jesús. (1999) "Entrenamiento temprano y captación de talentos en el deporte". Editorial INDE. Barcelona, España. Pág. 219 -230.

Matsudo, Victor, (2000) K. R. "New aproaches to talent detection". Sao Paulo, Brasil.

MATTO, M. (1977). "Talent search and development". Modern Athlete and Coach.

Memorias seminario internacional de softbol. (1996) "Confederación Panamericana de Sóftbol. Curso de superación técnica profesional". Medellín, Colombia.

Ministerio de educación y ciencia. (Año) "Test Europeo de Aptitud Física - EUROFIT Traducción de la edición en castellano": Mercedes Edo. Madrid, España.

Morales, Antonio. (2009) "Presidente Federación de Sóftbol de Colombia. Informe documental Cartagena".

Nadori, L. (1983) "El talento y su selección. Algunos problemas teóricos y metodológicos de la selección de talento deportivo". Revista Di cultura Sportiva. No 28, 29. Pág. 101 - 108.

Navarro, F. (2008) "Detección de talentos: realidad o mentira. En detección de talentos y la búsqueda de la excelencia en el deporte". Toledo, España.

Perdono, Luis. (2008) "Manual del Entrenador de Sóftbol". Impreso en los talleres de Graficas Uregon, C.A. Caracas, Venezuela.

Simpson, B, y Bristow, C. (1989) "Entrenamiento de Softbol Nuevo y Mejorado. J. O. Publications. Primera publicación en español. Asociación de Softbol Aficionado" (Amateur Softball Association) ASA. Estados Unidos.

Softbol basico. (2003) "Fundamentos técnicos". Grupo de estudio Kinesis. Editorial Kinesis, Pág. 11 - 15.

Universidad de Antioquia. (1992) "Instituto universitario de Educación Física. Manual de técnicas para la toma de las medidas antropométricas según protocolo de medicina deportiva de la U. de A". Medellín. Pág. 8

Zatsiorski, V.M. (1998) "Metrología deportiva". Habana. Cuba. Edición Puebla y Educación. 